\title{
Are there correlations between attention, physical endurance and anthropometric parameters of athletes?
}

\author{
Mirosław Mikicin ${ }^{1}$, Sylwia Nowacka-Dobosz ${ }^{2}$, Anna Mróz ${ }^{3}$, Anna Kuk $^{4}$, Adriana Zagórska-Pachucka ${ }^{4}$ \\ 1 Interfaculty Laboratory of Neuropsychophysiology, Józef Piłsudski University of Physical Education, Warsaw, Poland; \\ 2 Department of Theory and Methodology of Physical Education, Józef Piłsudski University of Physical Education, Warsaw, \\ Poland; ${ }^{3}$ Department of Physiology and Sports Medicine, Józef Piłsudski University of Physical Education, Warsaw, \\ Poland; ${ }^{4}$ Department of Pedagogy and Psychology, Józef Piłsudski University of Physical Education, Warsaw, Poland
}

\section{Summary}

Study aim: The aim of the study was to investigate the relationship between attention and physical endurance (running) and anthropometric parameters of athletes.

Material and methods: The study examined 61 students aged 19 to 25 years, divided into two groups: athletes (33 participants) and non-athletes (28 participants). We employed anthropometric measurements and the Vienna System Test, including tools to measure focused attention, such as LVT (visual orientation performance test) and DAUF (test for examination of sustained attention) and the Cooper test to measure endurance.

Results: Analysis of the results demonstrated a relationship between attention and physical endurance with median time from LVT $(\mathrm{r}=-0.552)$. A relationship was also found between the Cooper test results and the mean time to incorrect answer $(\mathrm{r}=-0.900)$.

Conclusions: The analysis demonstrated a relationship between attention, physical endurance and anthropometric parameters of athletes.

Keywords: Sustained attention - Physical endurance - Anthropometric parameters - Cooper test - Athletes

\section{Introduction}

Studies on attention deficit $[1,4,9]$ have shown that the lack of sustained attention and focus of attention may be the basis for motor neglect. These authors argue that this is likely to be caused by the lack of dominance of the right hemisphere in terms of attention mechanisms needed for searching the environment. It has also been demonstrated that inability to maintain a motor attitude for a specific time, disturbed continuity of control over motor activity or susceptibility to distraction is always connected to a certain degree with mental degradation. Simon et al. [14] demonstrated that, although programming of conscious and purposive motor activity is the precondition for performing this activity, it must also be supported by the functions of attention and perception. These functions help the person see objects located in space, feel the need for changing body position, etc. Consequently, this allows for processing of exteroceptive sensory information organized into spatial coordinates of the environment and planning of the movement direction. Although the mechanism of attention is more complex [3, 12, 14], it includes the increase in physiological reactivity, preparation for action and selectivity of reaction. As mentioned before, motor neglect (and the related poor physical endurance) is mostly observed in the case of the lack of dominance of the right cerebral hemisphere [3,11], although this dysfunction may also occur if the left hemisphere of the brain is damaged.

Since attention is considered to be the mechanism of reduction in information and the focus of attention in a predictable or unpredictable manner on a specific activity, situation, object or phenomenon, it determines the accuracy and quality of various cognitive processes. An important property of attention is concentration, which consists in exclusion of events or objects which the person perceives as unneeded at a specific moment or neglecting 
insignificant stimuli. The moment of distraction or dissipation of mind during sports competition, especially in sports which require high precision of movements or maximal effort over a short time, may cause a substantial error. Problems of concentrations are currently considered as both a theoretical and a practical problem of sport psychology. According to Karageorghis and Terry [8], concentration is an overriding psychological skill. Different sports require different concentration styles. In team sports, such as handball, athletes are able to effectively switch between a wide and narrow range of concentration. They also show good awareness of place and time. However, it should be noted that athletes from the same sport may differ significantly in the level of concentration and its correlation with physical endurance.

In sport, physical endurance is of key importance due to the necessity of maintaining the rate of an athletic activity over a specific time. The assumption was made that a lack of physical endurance may be linked to a reduction in the level of vigilance (attention function) and the lack of maintaining the focus of attention and sustained attention. No mechanism has been explored to control this phenomenon to date. Solving this problem may provide new insights into the development of endurance in athletes. Although the investigations of motor neglect have been continued in the clinical area, no studies have explored the problem of motor neglect in sport. Therefore, the problem seems to be new in this field. The relationships between physical endurance and various morphological parameters (particularly body fat percentage) have been analysed for many years. A substantial decline in abilities to perform exercise has been found to be related to increased body mass and subcutaneous fat [10]. Numerous studies have demonstrated the relationships between lean body mass and certain functional indices, e.g. oxygen uptake, stroke volume and blood pressure. A significant correlation was also found between body fat and human motor abilities $[7,15]$.

The need arises for verification of previous views concerning attention and physical endurance and extending the knowledge about correlations of attention with motor abilities and various anthropometric parameters of athletes. The aim of this study is to evaluate the level of concentration, sustained attention and physical endurance in athletes. The research problem was to demonstrate differences in the above variables with respect to physical activity and gender and to search for correlations between them.

\section{Material and methods}

\section{Study participants}

The examinations were performed in the Interfaculty Laboratory of Neuropsychophysiology and in the
Department of Physiology and Biomedical Science and the Chair of Theory of Physical Education and Pedagogy of the University of Physical Education. Statistical and qualitative analyses were performed for the results obtained by individual people from the groups of athletes and non-athletes. Among 61 study participants, 33 people were athletes involved in team sports, whereas 28 people were non-athletes. All the participants were students of the University of Physical Education aged 19 to 25 years.

All the study participants performed the tests included in the Vienna Test Battery: DAUF and LVT [13], anthropometric measurements, measurements of morphological parameters and fat percentage and the Cooper test.

\section{Research tools}

Vienna Test Battery - (C) Dr. G. Schuhfried [13]

DAUF: Sustained attention test. The test measures sustained and selective attention over specified time intervals. Contrary to wakefulness, sustained attention is defined as a process of continuous updating of the memory of the stimuli which are constantly present in the observation field and are frequently repeated. On the other hand, the examination of wakefulness requires the response to stimuli which occur relatively rarely in time and space. Measurement of sustained attention focuses mainly on the aspect of the ability to perform or to be ready to perform a task, which is largely independent of the intelligence. The DAUF sustained attention test is a computer-based test included in the Vienna Test Battery used to evaluate long-term selective sustained attention. A series of five triangles (stimuli) pointing upwards or downwards are presented on the computer screen, in either regularly or irregularly spaced rows which are impossible to predict. The respondent must press the button on the control panel as soon as he or she sees the critical stimulus, i.e. two triangles oriented downwards. Six hundred stimuli are presented during the test, including 120 critical stimuli. The rows of triangles displayed on the screen are of the same size and shape. Triangles can be pointing either downwards or upwards. One row of triangles can be observed at a time. Depending on the selected parametric test, the time of displaying individual rows, the line on the screen where rows appear and the number of triangles in the row may differ. The task of the person is to respond as fast as possible (by pressing the respective button on the control panel) to the critical stimuli, which is the specified number of triangles pointing downwards. The test duration is 35 minutes [13].

LVT: Visual pursuit test. The LVT test is a method used to examine oriented visual perception. The linear tracking tests have been used mainly in the psychology of road traffic and transportation. This test can be used wherever a person is required to show selective attention and wherever much depends on visual perception. The LVT test represents a procedure of examination of the process of visual 
structuring and speed of visual information processing under conditions of tracking of simple optical structures displayed with the background of a relatively complex environment and under conditions of time pressure. The number of errors is considered as a measure of correctness of information processing whereas the time of individual answer is used to evaluate the processing speed. Examination of complex perception properties requires the use of specialized psychological procedures. In the past, the development of these procedures has often lacked any substantial theoretical basis, especially because their development occurred mainly through solving specific experimental or practical problems. The same pattern has been observed for linear tracking. The LVT test is not a new update of the previous procedure, but is based on the experiences and observations of the previous version. In particular, the test evaluates the aspects of perception and visual orientation which are examined during orientation of observation of simple optical structures in a relatively complex environment and the effect of disturbances and time pressure. The test starts from the instruction with exercises. If the respondent solves at least 6 of 8 exercises correctly, the test moves to the main phase. The working rate can be adjusted by the respondent individually. The test can be performed in either the long (S1) or the short form (S2). The following variables are measured: - performance time - number of correct answers - median time of correct answers (s) - median time of incorrect answers (s) - number of correct answers determined compared to time limit. The short form is ca. 15 minutes. Duration: ca. 25 minutes [13]

Physical endurance test - the Cooper test. The Cooper test is an endurance test, developed by Cooper [5], consisting in a 12-minute continuous run. Currently, it is widely used to examine physical endurance, especially of athletes. Cooper [5, 6] developed a set of exercises including running, swimming and cycling. The running test has gained the most popularity. The test assumes that physical endurance, depending on age and gender, can be determined based on the distance covered over 12 minutes of running. People who are unable to run for 12 minutes continuously are allowed to walk to complete the test or, after resting by walking, return to running during the test at any time. The main rule is to perform 12 minutes of continuous physical exercise. It is recommended that the circumference of the circular running track on which the test is performed should be previously measured. If the track is unavailable in local facilities, an even route with marked $50 \mathrm{~m}$ sections should be prepared. A stopwatch is needed for time measurement. The respondents who participate in the test should be previously prepared for such an exercise through a cycle of physical exercise classes. Only people with good health status can participate in the test. A warm-up should be administered before the test. Several people can perform the test simultaneously. At a starting signal, the participants start running and the supervisor announces the time remaining to completion of the test every minute (e.g. "11 minutes to go!", "10 minutes to go!" etc.) and his or her assistance counts and records the laps completed by individual runners. After 12 minutes, the supervisor announces the completion of the test. At this signal, all the participants must stop running and wait until the assistants record the result. The test is performed once. The number of metres represents the test result. The test was chosen for several reasons. It is relatively easy to perform, highly correlated with the level of physical endurance and its duration is relatively long, similar to the duration of the measurements of attention and concentration.

Anthropometric measurements. Body height measurements were performed using the stadiometer with the accuracy of $0.1 \mathrm{~cm}$, body mass was measured with the accuracy of $0.1 \mathrm{~kg}$, whereas body composition was evaluated using bioelectrical impedance methodology by means of a Tanita BC 41 MA analyser. Body composition was analysed based on the measurement of tissue resistance measurement during the flow of electrical current with intensity of $<1 \mathrm{~mA}$ through the human body. The values of the impedance were used to evaluate fat percentage (FAT\% and $\mathrm{kg}$ ), lean body mass (LBM\% and $\mathrm{kg}$ ) and total body water (TBW\% and $\mathrm{kg}$ ). The body mass index (BMI) was also calculated.

\section{Statistical analysis of the data}

The means for each test and anthropometric measurements and the differences in the indices of aerobic and anaerobic capacity measured during the first and the second test were evaluated by means of Student's t-test for dependent samples. Non-parametric tests were used for the statistical data analysis: the Wilcoxon signed-rank test, the Mann-Whitney U-test, Spearman's R correlations and descriptive statistics.

\section{Results}

Table 1 presents mean values of individual anthropometric indices, body components and results of the Cooper test of both female and male athletes and non-athletes. The analysis showed that female non-athletes were shorter than men and were characterized by lower body mass and lower lean body mass compared to men. Fat percentage was significantly higher in women and maintained within the upper range of the standard for this age group, especially among athletes. BMI does not differentiate between study participants, which is consistent with the suggestions present in the literature that the index can distort the evaluation of fat percentage in athletes. Differences in the Cooper test between the groups with respect to gender 
Table 1. Mean values of anthropometric parameters and body composition and the Cooper test in the groups of athletes and non-athletes divided according to gender $(\mathrm{N}=61)$

\begin{tabular}{lcccc}
\hline Variables & Female athletes & Male athletes & Female non-athletes & Male non-athletes \\
\hline Body height $[\mathrm{cm}]$ & $174.98 \pm 6.81$ & $181.20 \pm 8.49$ & $167 \pm 2.24$ & $182.56 \pm 7.83$ \\
Body mass $[\mathrm{kg}]$ & $70.61 \pm 8.22$ & $82.44 \pm 11.81$ & $59.76 \pm 9.15$ & $76.77 \pm 6.03$ \\
BMI & $23.09 \pm 2.81$ & $25.44 \pm 3.89$ & $21.36 \pm 2.83$ & $23.07 \pm 1.46$ \\
FAT \% & $25.9 \pm 5.09$ & $15.16 \pm 7.36$ & $22.54 \pm 5.72$ & $10.67 \pm 4.1$ \\
FAT $[\mathrm{kg}]$ & $18.6 \pm 5.6$ & $13.60 \pm 7.72$ & $13.93 \pm 5.52$ & $8.12 \pm 2.98$ \\
FFM \% & $74.1 \pm 5.09$ & $84.83 \pm 7.36$ & $77.46 \pm 5.72$ & $89.33 \pm 4.1$ \\
FFM k[g] & $52.02 \pm 4.09$ & $68.83 \pm 7.88$ & $45.84 \pm 3.83$ & $68.64 \pm 6.93$ \\
TBW \% & $54.25 \pm 3.72$ & $61.50 \pm 5.07$ & $56.71 \pm 4.2$ & $65.42 \pm 3$ \\
TBW [kg] & $38.09 \pm 3$ & $50.38 \pm 5.77$ & $33.57 \pm 2.82$ & $50.26 \pm 5.08$ \\
Cooper test [m] & $2516 \pm 394.47$ & $2643 \pm 323.95$ & $2281 \pm 408.48$ & $2336 \pm 315.63$ \\
\hline
\end{tabular}

are not statistically significant. Furthermore, differences in endurance are observed between the athletes and nonathletes in favour of the former. Table 1 illustrates the significant differences in fat percentage and lean body mass between athletes and non-athletes. It should be emphasized that visual assessment of both female and male nonathletes seems to support the notion of their "slenderness" compared to athletes.

Table 2 presents means and standard deviations concerning concentration of attention (total of correctly performed tasks and mean time to take a proper decision) for the group of athletes. The total of correctly performed tasks is used as a measure of accuracy of the test.

Concerning the number of correct answers, the group of athletes was worse than the non-athletes. Time of correct answers was shorter in the case of athletes compared to the non-athletes, which can point to higher reaction impulsiveness and readiness of the nervous system in the athletes. This result can suggest that athletes perceive and respond to stimuli faster than those who are untrained. Their reaction times are similar both for correct and incorrect stimuli. Non-athletes tend to need more time to make a decision at the moment of the appearance of the incorrect stimuli. The examinations suggest that athletes are oriented towards searching for tasks consistent with the instruction and the answers to these tasks take less time than in the case of non-athletes. However, the speed of their reaction also translates into a higher number of mistakes made during task solving compared to non-athletes. In the case of tasks inconsistent with the instruction, reaction time in

Table 2. Differences in attention between athletes and non-athletes $(\mathrm{N}=61)$

\begin{tabular}{lcccc}
\hline Test & Group & $\mathrm{N}$ & $\mathrm{M} \pm \mathrm{SD}$ & $118.42 \pm 3.51$ \\
\hline DAUF, total correct & athletes & 33 & $118.39 \pm 1.61$ \\
& non-athletes & 28 & $3.15 \pm 3.26$ & $1.71 \pm 1.92$ \\
DAUF, total incorrect & athletes & 33 & 28 & $0.58 \pm 0.3$ \\
& non-athletes & 33 & $0.74 \pm 0.14$ \\
DAUF, mean time of correct & athletes & 28 & $0.81 \pm 0.34$ \\
& non-athletes & 31 & $0.8 \pm 0.15$ \\
DAUF, mean time of incorrect & athletes & 28 & $17.27 \pm 1.52$ \\
& non-athletes & 33 & $18 \pm 0.47$ \\
LVT: number of correct answers & athletes & 28 & $3.92 \pm 1.56$ \\
LVT: median of times of correct answers & non-athletes & 33 & $3.67 \pm 0.52$ \\
\hline
\end{tabular}

\footnotetext{
$* *-\mathrm{p}<0.001, *-\mathrm{p}<0.05$.
} 
Table 3. Differences in attention between women and men $(\mathrm{N}=61)$

\begin{tabular}{|c|c|c|c|c|}
\hline Test & Gender & $\mathrm{N}$ & $\mathrm{M} \pm \mathrm{SD}$ & $\mathrm{p}$ \\
\hline \multirow{2}{*}{ DAUF, total correct } & women & 27 & $118.29 \pm 1.89$ & 0.65 \\
\hline & men & 34 & $118.5 \pm 3.35$ & \\
\hline \multirow{2}{*}{ DAUF, total incorrect } & women & 27 & $2.77 \pm 3.09$ & 0.47 \\
\hline & men & 34 & $2.26 \pm 2.57$ & \\
\hline \multirow{2}{*}{ DAUF, mean time of correct } & women & 27 & $0.70 \pm 0.24$ & 0.72 \\
\hline & men & 34 & $0.62 \pm 0.25$ & \\
\hline \multirow{2}{*}{ DAUF, mean time of incorrect } & women & 27 & $0.70 \pm 0.3$ & 0.65 \\
\hline & men & 34 & $0.72 \pm 0.38$ & \\
\hline \multirow{2}{*}{ LVT: number of correct answers } & women & 27 & $17.88 \pm 0.57$ & $0.03 *$ \\
\hline & men & 34 & $17.38 \pm 1.93$ & \\
\hline \multirow{2}{*}{ LVT: median of times of correct answers } & women & 27 & $4.01 \pm 1.67$ & 0.17 \\
\hline & men & 34 & $3.64 \pm 0.6$ & \\
\hline
\end{tabular}

$*-\mathrm{p}<0.05$.

athletes was longer than this time in the group of non-athletes. It should be noted that the time for athletes to make decisions was similar for both tasks solved correctly and incorrectly. In untrained people, this time was longer when making correct decisions and shorter when making incorrect decisions. The results obtained in the study suggest that the willingness to solve the test as soon as possible is the dominant motivation in the group of athletes, whereas the non-athletes wanted to perform the test correctly.

Analysis of median reaction time showed that nonathletes need more time to decide whether the answer is incorrect, whereas they make a relatively fast decision on the correct answers. In non-athletes, time spent for correct answers was similar to the time documented for the non-athletes. However, the athletes make the incorrect decisions faster than the untrained controls. Comparison of the results obtained in the groups of women and men revealed (Table 3 ) a significant difference in the level of concentration of attention, with better results observed in women $(\mathrm{p}<0.03)$.

Table 4 presents a comparison of the mean results (total of correctly performed tasks and mean time to make a correct decision) for the group of male athletes and non-athletes. The total of correct answers is used to

Table 4. Differences in attention between athletes and non-athletes (men) N=34

\begin{tabular}{|c|c|c|c|c|}
\hline Test & Group & $\mathrm{N}$ & $\mathrm{M} \pm \mathrm{SD}$ & $\mathrm{p}$ \\
\hline \multirow{2}{*}{ DAUF, total correct } & athletes & 16 & $118.2 \pm 4.79$ & 0.35 \\
\hline & non-athletes & 18 & $118.73 \pm 1.62$ & \\
\hline \multirow{2}{*}{ DAUF, total incorrect } & athletes & 16 & $3.2 \pm 2.95$ & 0.15 \\
\hline & non-athletes & 18 & $1.52 \pm 2.01$ & \\
\hline \multirow{2}{*}{ DAUF, mean time of correct } & athletes & 16 & $0.5 \pm 0.33$ & $0.00 * *$ \\
\hline & non-athletes & 18 & $0.71 \pm 0.12$ & \\
\hline \multirow{2}{*}{ DAUF, mean time of incorrect } & athletes & 16 & $0.66 \pm 0.53$ & $0.00 * *$ \\
\hline & non-athletes & 18 & $0.78 \pm 0.14$ & \\
\hline \multirow{2}{*}{ LVT: number of correct answers } & athletes & 16 & $16.66 \pm 2.76$ & $0.00 * *$ \\
\hline & non-athletes & 18 & $17.94 \pm 0.4$ & \\
\hline \multirow{2}{*}{ LVT: median of times of correct answers } & athletes & 16 & $3.67 \pm 0.7$ & 0.63 \\
\hline & non-athletes & 18 & $3.63 \pm 0.53$ & \\
\hline
\end{tabular}

$* *-\mathrm{p}<0.01$ 


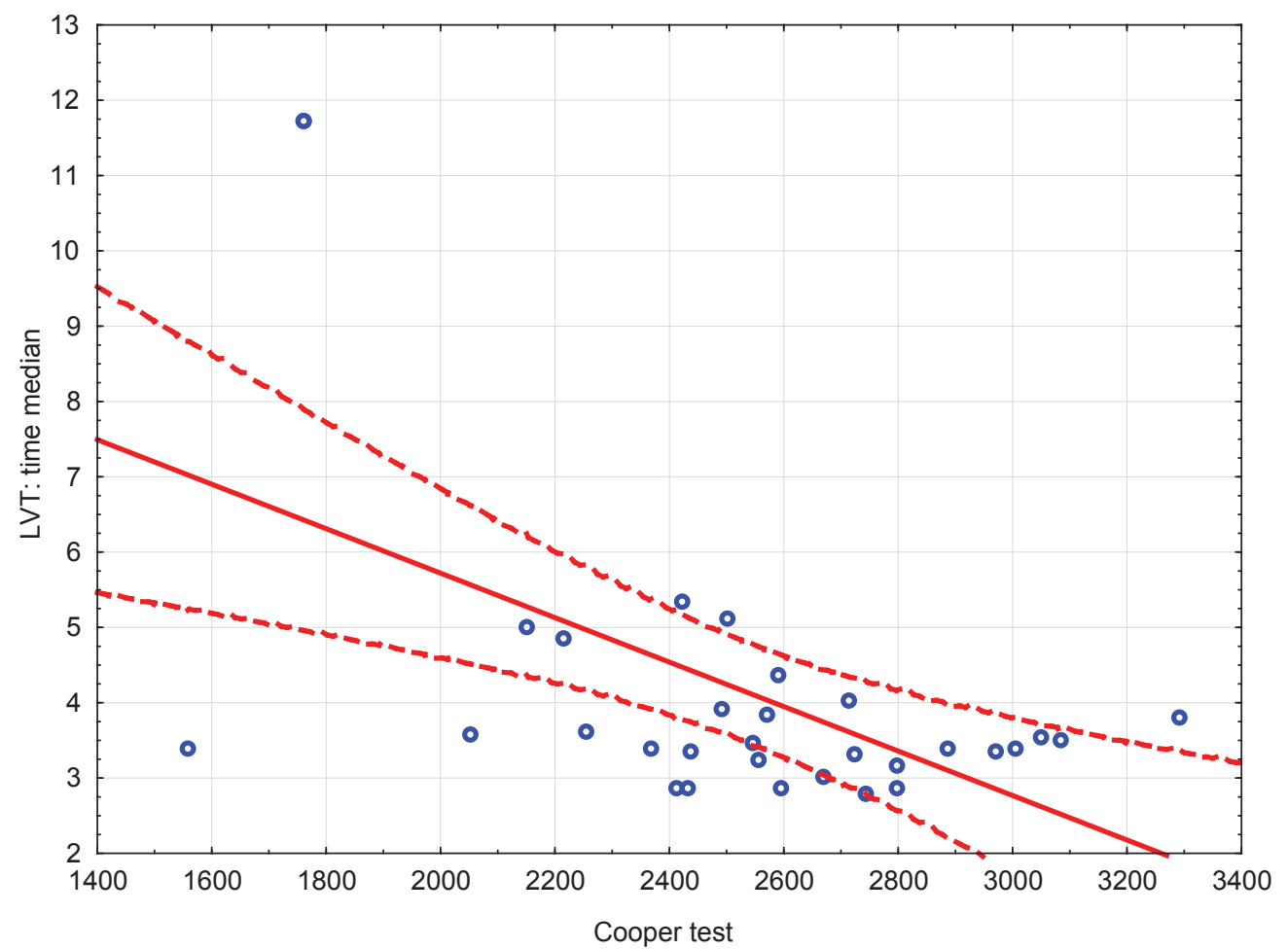

Fig. 1. Analysis of Spearman's correlation coefficients, Cooper test negatively correlated with median time for LVT $(r=-0.552)$ in athletes (women) $\mathrm{N}=27$

measure the accuracy of performing the test and relates to the abilities to select a specific stimulus and sustain attention on the task for a long time. No significant differences are observed between athletes and non-athletes with respect to the variable presented. The male participants differed in terms of correct answers, whereas athletes scored worse times compared to the non-athletes and did not differ much between each other in terms of correct reactions.

Comparison of female athletes and female non-athletes did not show substantial differences in attention.

Analysis of Spearman's correlation coefficients (Fig. 1) revealed correlations between attention and the Cooper test results at $p \leq 0.05$. The results of the Cooper test were negatively correlated with median time for LVT $(\mathrm{r}=-0.552)$ only in female athletes. A correlation was also found between the Cooper test results and the mean time to incorrect answer $(\mathrm{r}=-0.900)$.

\section{Discussion}

The examinations demonstrated that athletes are oriented towards searching for tasks consistent with the instruction and the answers to these tasks take slightly less time than in the case of non-athletes. However, the speed of their reaction also translates into a higher number of mistakes made during task solving compared to non-athletes. In the case of tasks inconsistent with the instruction, reaction time in athletes was longer than this time in the group of non-athletes. It should be noted that the time for athletes to make decisions was similar for both tasks solved correctly and incorrectly. In untrained people, this time was longer when making correct decisions and shorter when making incorrect decisions. The results obtained in the study suggest that the willingness to solve the test as soon as possible is the dominant motivation in the group of athletes, whereas the non-athletes wanted to perform the test correctly. This result is likely to suggest that non-athletes demonstrated a lower level of motivation connected with performing the task compared to non-athletes.

The consecutive results obtained in the study showed that the negative correlation of the Cooper test results with BMI is also present with respect to other human psychomotor abilities [2]. Furthermore, the studies tend to use the indices for which human endurance abilities are expressed by indices of aerobic capacity per body mass or per lean body mass. Endurance changes significantly in successive decades of human life. The results obtained for correlations in both groups suggest an average level of abilities connected with selected and sustained visual perception in the study group and the number of correctly solved tasks over a limited time. 


\section{Conclusions}

The analysis demonstrated a relationship between attention, physical endurance and anthropometric parameters of athletes. The Cooper test results were negatively correlated with median time in female athletes. A relationship was also found between the Cooper test results and the mean incorrect answer.

Conflict of interest: Authors state no conflict of interest.

\section{References}

1. Bartolomeo P., Chokron S. (2002) Orienting of attention in left unilateral neglect. Neurosci. Biobehav. Rev., 26: 217-234.

2. Beunen G., Malina R.M., Ostyn M., Renson R., Simons J., Van Gerven D. (1983) Fatness, growth and motor fitness of Belgian boys 12 through 20 years of age. Hum. Biol., 55: 599-613.

3. Buxbaum L.J., Ferraro M.K., Veramonti T. (2004) Hemispatial neglect. Subtypes, neuroanatomy, and disability. Neurology, 62: 749-756.

4. Corbetta M., Kincade M.J., Lewis C., Snyder A.Z., Sapir A. (2005) Neural basis and recovery of spatial attention deficits in spatial neglect. Nat. Neurosci., 8: 1603-1610.

5. Cooper K.H. (1978) The Aerobics Way. A Corgi Book, London.

6. Cooper K.H. (2003) Overcoming Hypertension. Bantam Books, New York.

7. Doherty D., Gaul C. (1991) Relationship of body size, physique and composition to physical performance In Young boys and girls. Int. J. Sport Med., 12(6): 525-532.
8. Karageorghis C.I., Terry P.C. (2011) Inside Sport Psychology. Human Kinetics, Champaign, USA.

9. Kessel M.E., Nes I.J., Brouwer W.H., Geurts A.C., Fasotti L. (2010) Visuospatial asymmetry and non-spatial attention in subacute stroke patients with and without neglect. Cortex, 46(5): 602-612. DOI: 10.1016/j. cortex.2009.06.004.

10. Malina R.M., Bouchard C. (1991) Growth, maturation and physical activity. Human Kinetics Books, Champain II.

11. Na D.L., Adair J.C., Choi S.H., Seo D.W., Kang Y., Heilman K.M. (2000) Ipsilesional Versus Contralesional Neglect Depends on Attentional Demands, 455-467.

12. Posner M.I., Gilbert C.D. (2000) Attention and primary visual cortex. Proceedings of the National Academy of Sciences USA, 96(6): 2585-2587.

13. Schuhfried G. (2004) Vienna-Test-System. www.schuhfried.com.

14. Simon E.S., Hegarty A.M., Mehler M.F. (1995) Hemispatial and directional performance biases in motor neglect. Neurology, 45: 525-531.

15. Toth M., Gardner A., Ades P. (1994) Contribution of body composition and physical activity to age-related decline In peak Vo2 in men and women. J. App. Phys., 77(2): 647-652.

\section{Received 06.03.2019 \\ Accepted 29.07.2019}

(C) University of Physical Education, Warsaw, Poland

\section{Acknowledgments}

This study was financed from budgetary funds of the Ministry of Science and Higher Education in Poland on science in 2014-2015. 